EL CERTIFICADO DE DEPÓSITO A TÉRMINO EN COLOMBIA: ¿INSTRUMENTO DE AHORRO O DE INVERSIÓN? ${ }^{1}$

\title{
THE TERM DEPOSIT CERTIFICATE IN COLOMBIA: ¿A SAVING OR AN INVESTMENT INSTRUMENT?
}

\author{
José Rodrigo Catica ${ }^{2}$, Óscar Hernán López y Mercedes Parra Alviz ${ }^{4}$ \\ Universidad de Tolima (Colombia)
}

RECIBIDO: Agosto 14 de 2014

ACEPTADO: Noviembre 05 de 2014

\section{RESUMEN}

El presente artículo es producto de una investigación cuyo objetivo consistió en analizar la verdadera naturaleza de los certificados de depósito a término en Colombia. La realidad de este instrumento financiero se descifra a partir del análisis de las tres rentabilidades propias a cualquier proceso de inversión. El tipo de investigación se da en un marco descriptivo en el cual se evidencian realidades del mercado y se sigue un método de investigación inductivo desde la observación sistemática de la realidad del instrumento de ahorro objeto de estudio. Se parte de la rentabilidad bruta que ofrecieron todos los establecimientos financieros a la población colombiana entre abril del año 2011 y marzo del año 2012 en los periodos de redención comúnmente manejados por la banca, los cuales corresponden a 30, 60, 90, 120, 180 y 360 días. Esta rentabilidad es sometida a los impuestos sobre los rendimientos financieros, denominados retención en la fuente, y posteriormente se descuenta el efecto inflacionario para terminar con la rentabilidad real. Se concluye que el certificado de depósito a término ofrece una rentabilidad negativa para los plazos o periodos de redención de 30 y 60 días, con lo cual se confirma que dicho certificado no se constituye ni en instrumento de ahorro y menos aún en instrumento de inversión durante esos plazos.

Palabras claves: CDT, ahorro, inversión, ganancias, bancarización.

\begin{abstract}
In the present article it is established the true nature of the term "Certificates of Deposit" in Colombia. The reality of this financial instrument is decrypted from the analysis of the three returns innate to any investment process. The departure point is the gross return that was offered to the Colombian population by all financial institutions through the period between April 2011 and March 2012, in redemption periods commonly handled by banks, which are the days $30,60,90,120,180$ and 360 of the year. This return is subject to taxes on financial returns, named "Withholding taxes" and subsequently it is deducted the inflationary effect, ending with the real return, which appears in a negative form for redemption deadlines or periods of 30 and 60 days, thus confirming that in these periods the Certificate of Deposit does not constitute a saving instrument much less an investment instrument.
\end{abstract}

Key words: Saving, Investment, Profitability, CDT, banking

\section{Este artículo se puede referenciar}

Catica, J., López, O. \& Parra, M. (2015). Certificado de depósito a término en Colombia: ¿instrumento de ahorro o de inversión?, en Desarrollo Gerencial Revista de la Facultad de Ciencias Económicas, administrativas y contables de la Universidad Simón Bolívar 7(1), 160-174.

${ }^{1}$ Proyecto de Investigación: Certificado de depósito a término en Colombia: ¿instrumento de ahorro o de inversión.

${ }^{2}$ Magister en Administración. Docente TC Universidad del Tolima. Colombia. rodrigo.catica@hotmail.com

${ }^{3}$ Magister en Administración. Docente TC Universidad del Tolima. Colombia. ohlopezm@ut.edu.co

${ }^{4}$ Magister en Administración. Docente Universidad del Tolima. Colombia. mparra@ut.edu.co 


\section{1.- INTRODUCCIÓN}

La bancarización en Colombia se viene desarrollando muy lentamente, por la vía del consumo y no precisamente a través del ahorro, a pesar de ser el último la base de un sano proceso de este tipo. Saiden (2009:15) afirma: "Es oportuno precisar que generalmente se entiende por bancarización el acceso que tienen los agentes económicos al crédito; sin embargo, se trata de un concepto mucho más complejo" Algunos de los argumentos para no ahorrar en las entidades financieras obedecen a la carencia de estímulos por parte de estos establecimientos. Catica \& Parra (2012:7) afirma:

La baja bancarización es uno de los tantos impedimentos para el desarrollo de algunos países de América latina. [(...) Esta situación] se atribuye a causas como el entorno tributario del sector bancario con el impuesto del 4 x1000, la tasa de usura y desde luego el costo de los servicios financieros, que más ha tomado fuerza entre un gran número de analistas, autoridades económicas y, por supuesto, la población colombiana.

Sin embargo, ahondando en la realidad, este artículo presenta un análisis detallado de las verdaderas rentabilidades de uno de los tantos instrumentos de ahorro y/o inversión que ofrecen las instituciones financieras en Colombia. La pregunta problema deberá responder al interrogante: ¿Es el certificado de depósito a término en Colombia un instrumento de ahorro o de inversión? El objetivo de la investigación se concentra en identificar si el CDT en Colombia es un instrumento de ahorro o de inversión.

Para lograr el objetivo general, se identifican las instituciones del sistema financiero que captan recursos por la modalidad de CDT, se cuantifica la rentabilidad en términos efectivos que cada institución ofrece y posteriormente se calcula la rentabilidad real que estos instrumentos ofrecen a los clientes, para identificar si verdaderamente funcionan como instrumentos de ahorro y/o inversión.

Muchos autores han escrito sobre el sistema financiero, su evolución, su presente y las perspectivas Toro et al. (2012), pero no todos han enfatizado en la realidad de las captaciones que este realiza. Sin embargo, Cano et al. (2008) desarrollan un análisis para establecer una relación de causalidad entre la tasa de referencia (subasta de expansión), la tasa interbancaria y la tasa de interés de los CDT a 90 días (con frecuencia diaria) y Albea (2002) compara las fortalezas y debilidades de los certificados de depósito entre 1972 у 1998.

Melo \& Becerra (2006) establecen la relación entre dos tasas de interés de corto plazo, la CDT y la TIB y la tasa de subasta de expansión (SEXP), en el periodo enero de 2001- septiembre de 2005, con lo cual se identifica el interés en evaluar el rendimiento real de los CDT. 


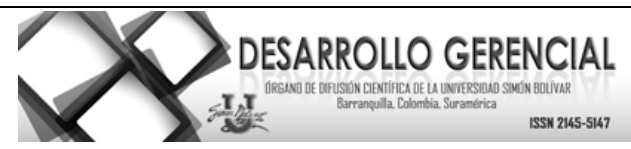

En el estudio se incorporan variables de medición de rentabilidad, así como la tasa de inflación y la tasa de impuesto de retención en la fuente. El estudio se limita a resultados históricos y su alcance corresponde a calificar el tipo de instrumento de captación de recursos del sistema financiero conocido como CDT. Pues existen diversas opiniones frente al rendimiento real de este sistema de captación, considerado inversión operativa; que requiere de seguridad y en especial, de rentabilidad, Villegas \& Ortega (1997).

Es necesario dejar en claro cuál es la rentabilidad real que otorgan los certificados de depósito a término en Colombia. Pocos conocen el rendimiento real, y aun cuando las definiciones han aportado a la realidad de un ahorro, no así a su verdadera rentabilidad. Baena et al (2008:7) afirman: "Técnicamente se considera que el ahorro es la base de capital, es decir, de las inversiones para conseguir rendimientos futuros" y esta situación es discutible en el caso colombiano.

Se hace necesario este tipo de análisis, ya que tradicionalmente las instituciones financieras han desarrollado una nueva gama de instrumentos financieros que ofrecen múltiples oportunidades de ahorro, pero ni siquiera este innovador y diversificado sistema de banca financiera ha sido capaz de atraer a la población alejándola de los métodos tradicionales de ahorro, Niculescu-Aron (2012). Por ello, estos nuevos métodos que no han convencido a los ahorradores por su rentabilidad merecen análisis incluso en el contexto internacional. Así, Muhleisen (1997) evalúa las tendencias del ahorro en India, sobre todo en los determinantes del ahorro y las estrategias para el ahorro. Mientras tanto Orazio et al. (2000) presentan un análisis descriptivo de las correlaciones de largo y de corto plazo entre el ahorro, la inversión y el crecimiento de las tasas de 123 países durante el periodo 1961-1994. Manteniendo el enfoque, Nokuman (2002) efectuó un análisis en modelos de tipos de interés a corto plazo en certificados de depósito japoneses.

En el desarrollo del estudio se demuestra que bajo algunas circunstancias contractuales la rentabilidad real del certificado de depósito a término lo descalifica como instrumento de ahorro y de inversión. Se aborda un análisis hasta hoy renuente para las instituciones financieras y desconocido para la población colombiana, que lamentablemente evidencia una cultura de baja formación financiera.

Este análisis permitió evidenciar que la modalidad de ahorro a través del certificado de depósito a término no garantiza ser en todos los casos tal instrumento y menos aún un instrumento de inversión, pues el efecto de los impuestos, y en especial de la inflación, lo dejan en la posición de instrumento destructor de valor para el ahorrador o para quien lo toma como un mecanismo de inversión. Dicha situación genera alta preocupación, por cuanto no se contribuye a los procesos de crecimiento de la economía tal y como lo plantea Arenas (2004:5): "Para lograr la meta de crecimiento, habrá que incrementar sustancialmente el 


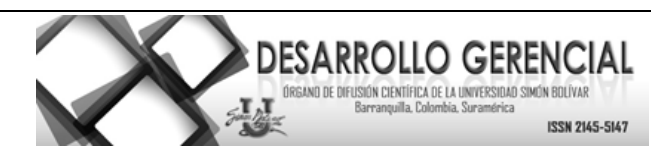

ahorro interno. Hay que regresar a tasas de ahorro, en relación con el PIB, del 20 por ciento o más, y saber invertir inteligentemente ese ahorro"

\section{2.- MÉTODO}

El tipo de investigación se enmarca en un proceso descriptivo en el cual se evidencian realidades del mercado y se sigue un método de investigación inductivo desde la observación sistemática de la realidad del instrumento de ahorro objeto de estudio. Se parte de la rentabilidad bruta que ofrecieron todos los establecimientos financieros a la población colombiana para el periodo comprendido entre abril del año 2011 y marzo del año 2012 en los tiempos de redención comúnmente manejados por la banca, los cuales corresponden a 30, 60, 90, 120, 180 y 360 días. Esta rentabilidad es disminuida por el impuesto sobre los rendimientos financieros, denominado retención en la fuente, y posteriormente se descuenta el efecto inflacionario, para terminar con la rentabilidad real.

Se tomó la información de todos los establecimientos que captan recursos por la modalidad de depósito a término y vigilados por la Superintendencia Financiera. El análisis se llevó a cabo teniendo en cuenta la rentabilidad neta y la rentabilidad real o final que ofrece el CDT, después de descontar los impuestos y el efecto inflacionario.

\section{Participantes}

Para efectos del presente estudio se han seleccionado todos los 23 bancos comerciales, 1 corporación financiera, 17 compañías de financiamiento comercial, 1 organismo cooperativo de grado superior, 2 instituciones oficiales especiales y 6 cooperativas financieras. Se tomó el total de instituciones financieras que ofrecen la modalidad de captación de recursos por certificado de depósito a término y que reportan información a la Superintendencia Financiera de Colombia.

\section{Instrumentos}

Se efectuó un proceso de revisión tanto de información secundaria como documental sobre comunicados oficiales de captación de recursos generados o divulgados por la Superintendencia Financiera de Colombia. Se toman estos informes por su veracidad y confiabilidad en la medida que son reportes oficiales de las instituciones financieras a su máximo organismo de vigilancia y control. 


\section{Procedimientos}

El proceso de investigación se inicia identificando las distintas instituciones financieras que desarrollan procesos de captación por la modalidad de depósito de ahorro a término ya mencionadas anteriormente. Se tomaron luego las rentabilidades brutas, en términos de tasa efectiva anual, que ofrecieron estos establecimientos y que fueron reportadas a la Superintendencia Financiera de Colombia. Estos rendimientos se ponderaron por volúmenes de captación y se promediaron para obtener una tasa bruta de rentabilidad promedio ponderada de todo el sistema financiero colombiano.

Para identificar el papel real del certificado de depósito a término (CDT) en la economía colombiana, y en especial en el proceso de bancarización que tanto se le reclama a las entidades financieras, se procede a tomar la información histórica de los últimos doce meses del comportamiento de la tasa efectiva anual de rentabilidad promedio ponderada de los certificados de depósito a término de todo el sistema financiero colombiano. Estas tasas de rendimiento, que son reportadas por cada institución a la Superintendencia Financiera, corresponden a los rendimientos que reconoce el sistema financiero colombiano a los depósitos de ahorro con una permanencia de 30, 60, 120, 180 y 360 días. Estos intervalos de tiempo son los plazos regulares a los cuales captan las instituciones financieras en Colombia y que para efectos del presente estudio se han tomado todos los 23 bancos comerciales, 1 corporación financiera, 17 compañías de financiamiento comercial, 1 organismo cooperativo de grado superior, 2 instituciones oficiales especiales y 6 cooperativas financieras.

A continuación la rentabilidad bruta o también denominada rentabilidad facial es sometida el efecto de los impuestos que por disposiciones formales se debe aplicar a los rendimientos financieros, y el cual corresponde al $7 \%$ o $10 \%$ sobre el $70 \%$ de los rendimientos percibidos, tal como se encuentra consagrado en el Estatuto Tributario colombiano. Al descontar el impuesto, se logra determinar la rentabilidad neta, que luego de ser sometida al efecto de la inflación para cada uno de los periodos de análisis establece cuál es la rentabilidad real para los dineros de los ahorradores y/o inversionistas que se mantienen en poder de la banca colombiana.

De esta manera se logra determinar si efectivamente estos dineros que se captan por medio de los instrumentos denominados en Colombia certificados de depósito a término (CDT), puedan recibir el calificativo de instrumento de ahorro o instrumento de inversión.

\section{3.- RESULTADOS}

Para medir la rentabilidad real de un instrumento financiero como el certificado de depósito a término, se debe iniciar el proceso de análisis con su rentabilidad bruta o rentabilidad facial, la cual corresponde a 
la tasa efectiva que ofrecen los distintos establecimientos e instituciones financieras, y que varía de acuerdo al plazo en que se pacta el depósito. Para efectos del presente estudio, se han tomado las tasas efectivas de rendimientos que se relacionan en la tabla 1, correspondientes a cada uno de los plazos mencionados anteriormente. Estas tasas comprenden al rendimiento de todo el sistema financiero representado en las instituciones antes mencionadas. (Tabla 1).

Tabla 1. Rendimientos efectivos por tiempo de ahorro

\begin{tabular}{|c|c|c|c|c|c|c}
\hline FECHA & $\begin{array}{c}\text { RENTABILIDAD } \\
\text { BRUTA 30 DíAS }\end{array}$ & $\begin{array}{c}\text { RENTABILIDAD } \\
\text { BRUTA 60 DÍAS }\end{array}$ & $\begin{array}{c}\text { RENTABILIDAD } \\
\text { BRUTA 90 DÍAS }\end{array}$ & $\begin{array}{c}\text { RENTABILIDAD } \\
\text { BRUTA 120 } \\
\text { DíAS }\end{array}$ & $\begin{array}{c}\text { RENTABILIDAD } \\
\text { BRUTA 180 } \\
\text { DÍAS }\end{array}$ & $\begin{array}{c}\text { RENTABILIDAD } \\
\text { BRUTA 360 } \\
\text { DÍAS }\end{array}$ \\
\hline abr-11 & $1,30 \%$ & $1,75 \%$ & $3,76 \%$ & $3,98 \%$ & $4,16 \%$ & $4,65 \%$ \\
\hline may-11 & $1,20 \%$ & $1,64 \%$ & $3,89 \%$ & $4,17 \%$ & $4,34 \%$ & $4,94 \%$ \\
\hline jun-11 & $1,43 \%$ & $1,46 \%$ & $4,12 \%$ & $4,37 \%$ & $4,53 \%$ & $5,29 \%$ \\
\hline jul-11 & $1,27 \%$ & $1,53 \%$ & $4,22 \%$ & $4,48 \%$ & $4,71 \%$ & $5,41 \%$ \\
\hline ago-11 & $1,27 \%$ & $1,44 \%$ & $4,50 \%$ & $4,63 \%$ & $4,86 \%$ & $5,82 \%$ \\
\hline sep-11 & $1,30 \%$ & $1,68 \%$ & $4,62 \%$ & $4,75 \%$ & $5,24 \%$ & $5,84 \%$ \\
\hline oct-11 & $1,22 \%$ & $1,68 \%$ & $4,72 \%$ & $5,05 \%$ & $5,29 \%$ & $5,91 \%$ \\
\hline nov-11 & $1,39 \%$ & $1,66 \%$ & $5,08 \%$ & $5,06 \%$ & $5,48 \%$ & $6,00 \%$ \\
\hline dic-11 & $1,34 \%$ & $1,80 \%$ & $5,13 \%$ & $5,31 \%$ & $5,53 \%$ & $6,21 \%$ \\
\hline ene-12 & $1,25 \%$ & $1,83 \%$ & $5,14 \%$ & $5,34 \%$ & $5,65 \%$ & $6,02 \%$ \\
\hline feb-12 & $1,40 \%$ & $1,86 \%$ & $5,29 \%$ & $5,49 \%$ & $5,77 \%$ & $6,18 \%$ \\
\hline mar-12 & $1,58 \%$ & $1,86 \%$ & $5,37 \%$ & $5,38 \%$ & & $5,86 \%$ \\
\hline
\end{tabular}

Nota: Superintendencia Financiera de Colombia $2011-2012$

En esta tabla se describe las rentabilidades brutas efectivas anuales promedio ponderadas de todo el sistema financiero colombiano para cada uno de los periodos.

Se puede apreciar que los rendimientos ofrecidos por los distintos establecimientos financieros dependen del plazo pactado al momento de constituir cada certificado de depósito a término. Es decir, en la medida en que el tiempo es mayor, así mismo las instituciones financieras incentivan al ahorrador con unas tasas un poco más altas que las otras ofrecidas a los depósitos en periodos inferiores. De la misma manera, se puede observar que los rendimientos vienen experimentando un leve crecimiento desde abril del año 2011 hasta marzo del año 2012, periodo al cual corresponde el objeto de estudio. Los rendimientos no varían mucho, por cuanto las variaciones normalmente corresponden a impactos de la política monetaria a largo plazo, Becerra \& Melo (2009:120).

En lo que respecta a los costos que merman la rentabilidad bruta del título y la convierten en la rentabilidad real, considerando los rendimientos financieros se puede observar que la tasa de retención en la fuente rendimientos financieros es un impuesto que no sufre modificaciones en el periodo de análisis y que, por el contrario, tiene su origen en el Decreto 16517/1961. Entre tanto, la tasa de inflación que re- 
expresa la rentabilidad neta en la verdadera rentabilidad o rentabilidad real, se comporta de manera contraria al crecimiento que muestra la tasa de rendimiento efectiva anual ofrecida por las instituciones financieras, véase tabla 2. Es decir, mientras la tasa de inflación mensual disminuye, la tasa de rendimiento experimenta leves incrementos.

Este fenómeno se puede explicar en parte porque la tasa que se relaciona corresponde a la inflación anual acumulada a la fecha, es decir, la inflación anual involucra el mes de apertura del título hasta su vencimiento, mientras que la tasa de rentabilidad tomada refiere a la fecha de apertura del título. (Tabla 2).

Tabla 2. Comportamiento de la tasa de inflación y la retención en la fuente

\begin{tabular}{|c|c|c|c|c|c|}
\hline Año/mes & $\begin{array}{c}\text { Índice de } \\
\text { precios al } \\
\text { consumidor }\end{array}$ & $\begin{array}{c}\text { Variación } \\
\text { mensual (\%) }\end{array}$ & $\begin{array}{c}\text { Variación año } \\
\text { corrido (\%) }\end{array}$ & $\begin{array}{c}\text { Variación anual } \\
\mathbf{( 5 )}\end{array}$ & $\begin{array}{c}\text { Retención en la } \\
\text { fuente }\end{array}$ \\
\hline 201204 & 110,92154 & $0,14 \%$ & $1,62 \%$ & $3,43 \%$ & $7,00 \%$ \\
\hline 201205 & 111,25436 & $0,30 \%$ & $1,92 \%$ & $3,44 \%$ & $7,00 \%$ \\
\hline 201206 & 111.34646 & $0,08 \%$ & $2,01 \%$ & $3,20 \%$ & $7,00 \%$ \\
\hline 201207 & 111,32241 & $-0,02 \%$ & $1,98 \%$ & $3,035 \%$ & $7,00 \%$ \\
\hline 201208 & 111,36807 & $0,04 \%$ & $2,03 \%$ & $3,115 \%$ & $7,00 \%$ \\
\hline 201209 & 111,68694 & $0,29 \%$ & $2,32 \%$ & $3,08 \%$ & $7,00 \%$ \\
\hline 201210 & 111,86942 & $0,16 \%$ & $2,48 \%$ & $3,06 \%$ & $7,00 \%$ \\
\hline 201211 & 111,71648 & $-0,14 \%$ & $2,34 \%$ & $2,77 \%$ & $7,00 \%$ \\
\hline 201212 & 111,81576 & $0,09 \%$ & $2,44 \%$ & $2,44 \%$ & $7,00 \%$ \\
\hline 201301 & 112,14896 & $0,30 \%$ & $0,30 \%$ & $2,00 \%$ & $7,00 \%$ \\
\hline 201302 & 112,64705 & $0,44 \%$ & $0,74 \%$ & $1,83 \%$ & $7,00 \%$ \\
\hline 201303 & 112,87881 & $0,21 \%$ & $0,95 \%$ & $1,91 \%$ & $7,00 \%$ \\
\hline
\end{tabular}

Fuente: Banco de la República 2012-2013

En esta tabla se describe el comportamiento de la retención en la fuente y la tasa de inflación medida por la variación anual en cada uno de los meses de estudio.

Otro aspecto que justifica en cierta medida esta situación, obedece a la presión que han ejercido distintos gremios, autoridades monetarias, organizaciones sociales y en especial organismos del Estado, en cabeza del Ministro de Hacienda, quien ha presionado de manera informal a los bancos para que incentiven el ahorro y mejoren los niveles de profundización financiera del país.

Con las variables que determinan el rendimiento real de un título valor, en este caso del certificado de depósito a término (CDT), se evalúa el comportamiento de las tres rentabilidades básicas de una inversión, y que corresponden a su rentabilidad bruta, rentabilidad neta y rentabilidad real. Esta última corresponde a la medición que debe ser objeto para toma de decisiones y que en Colombia infortunadamente queda relegada al interés prioritario de los ahorradores por la rentabilidad bruta, situación que en la gran mayoría de veces obedece al desconocimiento y la poca cultura financiera. 
Es evidente que la pretensión mínima en procesos de ahorro debe corresponder a que el titular del instrumento o del certificado de depósito a término, reciba un rendimiento o incentivo por permitir que sus recursos se incorporen en la dinámica del sistema financiero y que estos sean intermediados por los distintos establecimientos del sector. Sin embargo, esta pretensión mínima no se materializa en algunas condiciones que se describen en el presente estudio y mucho menos se da de manera masiva como se debiera presentar en una economía con altos niveles de profundización financiera; originado por la desconfianza que se tiene del sector bancario en Colombia, Cática at al (2014:10). El periódico Portafolio (2012), plantea que "En el 2011, el Banco de la República aumentó la tasa de intervención de 3 por ciento a 4,75. Esto hizo que el CDT sea considerado una alternativa de ahorro e inversión. Los depósitos en este instrumento crecieron 22 por ciento, al sumar 61,42 billones de pesos". Todo ello exige a las personas un conocimiento en temas financieros para mejorar los procesos de toma de decisiones Bernal (2012).

La transformación de la rentabilidad bruta de un depósito a término a 30 días hacia su rentabilidad real. Inicialmente se relaciona la rentabilidad bruta o rentabilidad facial, que corresponde a certificados de depósito a término constituidos desde abril del año 2011 hasta marzo del año 2012. (Tabla 3)

Tabla 3. Rentabilidad real del CDT en Colombia, año 2013-2013 para un plazo de 30 días.

\begin{tabular}{|c|c|c|c|c|c|c|}
\hline Fecha & $\begin{array}{c}\text { Tasa } \\
\text { promedio } \\
\text { ponderada }\end{array}$ & $\begin{array}{c}\text { Rentabilidad } \\
\text { bruta o facial }\end{array}$ & $\begin{array}{c}\text { Tasa de } \\
\text { impuestos }\end{array}$ & $\begin{array}{c}\text { Rentabilidad } \\
\text { neta }\end{array}$ & $\begin{array}{c}\text { Tasa de } \\
\text { inflación }\end{array}$ & Rentabilidad \\
\hline abr-12 & $1,30 \%$ & $1,30 \%$ & $7,0 \%$ & $1,21 \%$ & $3,43 \%$ & $-2,15 \%$ \\
\hline may-12 & $1,20 \%$ & $1,20 \%$ & $7,0 \%$ & $1,12 \%$ & $3,44 \%$ & $-2,25 \%$ \\
\hline jun-12 & $1,43 \%$ & $1,43 \%$ & $7,0 \%$ & $1,33 \%$ & $3,20 \%$ & $-1,81 \%$ \\
\hline jul-2013 & $1,27 \%$ & $1,27 \%$ & $7,0 \%$ & $1,18 \%$ & $3,03 \%$ & $-1,79 \%$ \\
\hline ago-12 & $1,27 \%$ & $1,27 \%$ & $7,0 \%$ & $1,18 \%$ & $3,11 \%$ & $-1,87 \%$ \\
\hline sep-12 & $1,30 \%$ & $1,30 \%$ & $7,0 \%$ & $1,21 \%$ & $3,08 \%$ & $-1,81 \%$ \\
\hline oct-2012 & $1,22 \%$ & $1,22 \%$ & $7,0 \%$ & $1,14 \%$ & $3,06 \%$ & $-1,86 \%$ \\
\hline nov-2012 & $1,39 \%$ & $1,39 \%$ & $7,0 \%$ & $1,29 \%$ & $2,77 \%$ & $-1,44 \%$ \\
\hline dic-2012 & $1,34 \%$ & $1,34 \%$ & $7,0 \%$ & $1,24 \%$ & $2,44 \%$ & $-1,17 \%$ \\
\hline ene-2013 & $1,25 \%$ & $1,25 \%$ & $7,0 \%$ & $1,16 \%$ & $2,00 \%$ & $-0,82 \%$ \\
\hline feb-2013 & $1,40 \%$ & $1,40 \%$ & $7,0 \%$ & $1,30 \%$ & $1,83 \%$ & $-0,52 \%$ \\
\hline mar-2013 & $1,58 \%$ & $1,58 \%$ & $7,0 \%$ & $1,47 \%$ & $1,91 \%$ & $-0,43 \%$ \\
\hline Promedios & & $1,33 \%$ & $7,0 \%$ & $1,24 \%$ & & $-1,49 \%$ \\
\hline
\end{tabular}

Fuente: Superfinanciera 2012-2013

En esta tabla se describe la tasa de captación promedio ponderada del sistema financiero para cada uno de los períodos de análisis, su respectiva tasa meta; una vez descontados los impuestos de ley y la tasa real final una vez incorporada la inflación. 


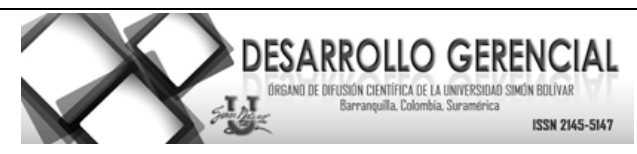

Estas rentabilidades brutas corresponden a los rendimientos ofrecidos por todos los establecimientos financieros y reportados a la Superintendencia Financiera de Colombia, los cuales se han promediado y ponderado por volúmenes de captación a fin de obtener una tasa bruta de rendimiento promedio ponderado de todo el sistema financiero colombiano. En el proceso de descontar los impuestos a la rentabilidad bruta o tasa bruta, se observa que el rendimiento neto para el ahorrador o inversionista queda significativamente por debajo de la rentabilidad facial o bruta. Esta rentabilidad neta se transforma en una rentabilidad real con signo contrario al que espera obtener cualquier ahorrador, por el efecto de la inflación.

Es claro que un ahorrador como mínimo espera mantener su dinero en términos nominales y desde luego desea que sus recursos no pierdan el poder adquisitivo, situación que en este caso no sucede, pues en todos los meses de análisis la tasa ofrecida en las entidades financieras colombianas es tan pequeña (por debajo de la inflación) que llevan a que el ahorrador pierda parte de sus recursos, por el efecto inflacionario.

La mayor pérdida de recursos se da en los certificados de depósito a término a 30 días que se constituyeron en el mes de abril del año 2011. Es decir, los títulos constituidos en esta fecha perdieron el $2,15 \%$ de su valor, y significa que esta modalidad de relación de la banca con la población colombiana no se puede considerar modalidad de ahorro y tampoco modalidad de inversión. Este procedimiento de análisis se repite en las modalidades de ahorro a término para los periodos de 60, 90, 120, 180 y 360 días. (Tabla 4).

Tabla 4. Resumen de rentabilidades por plazos año 2012-2013

\begin{tabular}{|c|c|c|c|}
\hline Período de inversión & Rentabilidad facial & Rentabilidad neta & Rentabilidad real \\
\hline 30 días & $1,33 \%$ & $1,24 \%$ & $-1,49 \%$ \\
\hline 60 días & $1,68 \%$ & $1,56 \%$ & $-1,17 \%$ \\
\hline 90 días & $, 4,66 \%$ & $4,33 \%$ & $1,52 \%$ \\
\hline 120 días & $4,83 \%$ & $4,50 \%$ & 1,68 \\
\hline 180 días & $5,12 \%$ & $4,76 \%$ & $1,94 \%$ \\
\hline 360 días & $5,72 \%$ & $5,32 \%$ & $2,48 \%$ \\
\hline
\end{tabular}

Fuente: Autores

En la tabla se resume el comportamiento de las tres rentabilidades para cada uno de los plazos objeto de estudio.

Si un ahorrador en Colombia depositó sus recursos en el último año con plazos entre 30 y 60 días, experimentó un deterioro real de sus ahorros porque los rendimientos que le ofreció la banca colombiana no fueron suficientes para absorber el efecto inflacionario. Desde esta óptica, el certificado de depósito a término en Colombia no es un instrumento de ahorro. Ahora bien, si un inversionista efectuó la misma 
operación, es decir, utilizó este instrumento financiero como modalidad de inversión, se encontró con una situación similar que permite afirmar que en los plazos mencionados (30 días y 60 días) y para las fechas objeto de análisis, el certificado de depósito a término no fue un instrumento de inversión, pues los rendimientos esperados no se ven materializados en el análisis; por el contrario, aquel experimentó la pérdida de una parte de su capital invertido. De ahí que la falta de productos atractivos en el sistema financiero, llevan a la proliferación de las llamadas pirámides

Sin embargo, las operaciones de ahorro o de inversión que se efectuaron en plazos de 90 días y superiores a estos, ofrecieron a los titulares del instrumento una rentabilidad real de muy bajos porcentajes si se comparan con los altos porcentajes que se vienen experimentando en el sistema financiero cuando se trata de recursos que colocan los establecimientos financieros en poder de sus clientes en calidad de préstamos.

Las rentabilidades brutas en los plazos de 30 y 60 días se encuentran por debajo de la línea de cero rentabilidad, con lo cual se ratifica que para estos plazos de redención de los certificados de depósito a término, este título no se constituye en instrumento de ahorro y menos aún en un instrumento de inversión para la población colombiana. (Figura 1)

Figura 1. "Resumen de rentabilidades por plazos (periodo abril 2012-marzo 2013)"

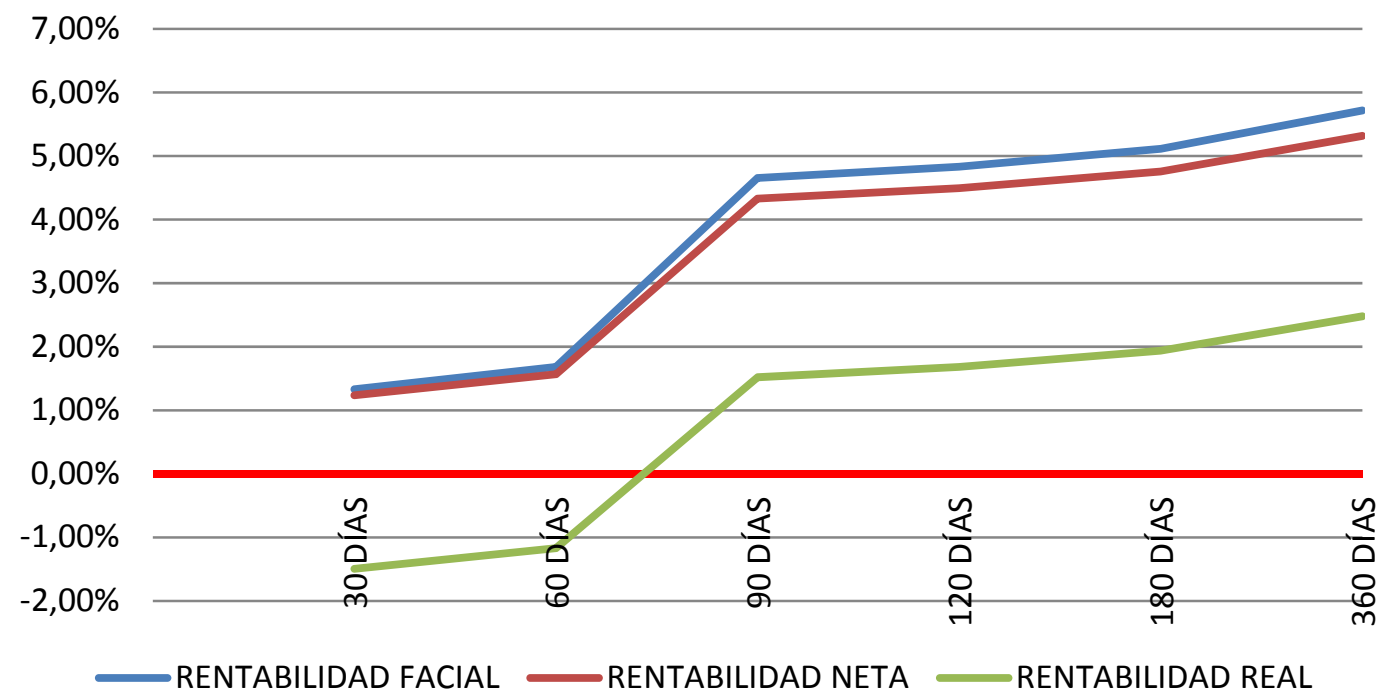

Fuente: Autores 


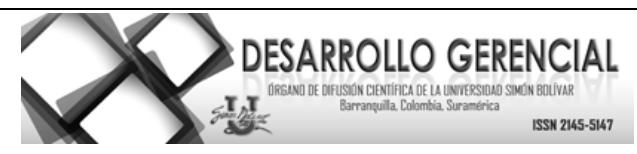

\section{4.- DISCUSIÓN}

El ahorro desde una concepción ligera se entiende como la diferencia entre los ingresos y los gastos, que se acumula con fines particulares. Esta acumulación terminará siendo simplemente ahorro o inversión de acuerdo al destino que la unidad económica tenga para los mismos. Por definición, el ahorro en una economía durante un periodo dado, es la parte del ingreso que no se consume. $\mathrm{O}$ sea que si en un cierto periodo de tiempo (usualmente se toma un año) el ingreso es $\mathrm{Y}$ y el consumo es $\mathrm{C}$, el ahorro es $\mathrm{S}=\mathrm{Y}-\mathrm{C}$.

Si se trata de una economía monetizada, el ahorro refiere a la formación de recursos financieros, cuyo destino es la adquisición de aquellos bienes de capital que mantienen en operación el aparato productivo, y asimismo son necesarios para ensanchar su capacidad y/o su eficiencia con el fin de obtener en el futuro un aumento en la producción de bienes y servicios, o sea, provocar el crecimiento económico, Poveda (1968:12).

"Toma importancia el ahorro en la medida en que este es insumo fundamental para los procesos de crecimiento de las economías" (Caja de Crédito Agrario Industrial y Minero, 1967:149), y existen innumerables medios y facilidades que ofrecer a quien desee colocar sus recursos. Para eso existen instrumentos financieros e instituciones financieras a las cuales puede una persona llevar el sobrante mensual de sus ingresos. Estas sumas depositadas van aumentando poco a poco, y al mismo tiempo ganando intereses, de tal forma que al final lo que la persona ahorra se convierte en capital relativamente importante para su bienestar. Sin embargo, este aspecto se desvirtúa un poco por los bajos intereses que vienen reconociendo hoy en día los establecimientos financieros y sobre lo cual las reclamaciones no se han hecho esperar.

Ante todo, los bancos son intermediarios entre deudores y acreedores, pero no en el sentido de tomar prestado de unos clientes para entregárselo a otro, sino más bien en el de que crean para sus prestatarios ciertos fondos, que estos podrán utilizar como capacidad adquisitiva real. El prestatario se convierte en deudor del banco, debido a que, para sus actividades particulares, puede utilizar la promesa del banco como un equivalente de numerario. A su vez, por las obligaciones que contrae con sus depositantes, el banco se convierte en deudor de cualquier persona que designe el prestatario. El hecho de que este último satisfaga intereses mientras que el banco contrae una obligación que no exige el abono de intereses, o que implica, en todo caso, unos intereses relativamente reducidos, explica el porqué de los beneficios que se derivan de las actividades bancarias.

Esta argumentación corresponde a la explicación más clara del por qué los volúmenes de ahorro para el año 2012 tan solo crecieron el 5\%, mientras el consumo representado en las tarjetas de crédito crecieron al $8 \%$. Los pocos intereses no son incentivo para el ahorro y además, contribuyen a las abultadas utilidades 


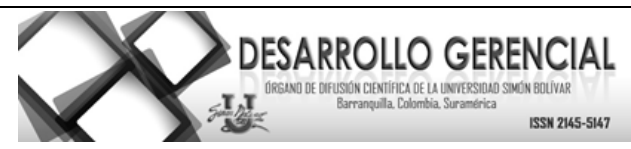

del sector financiero. Kasilingam \& Jayabal (2009) lograron identificar la percepción de los inversionistas frente a cada tipo de inversión y ahorro.

Según Parra (1976:30), como primera medida, los países en desarrollo que pretendan alcanzar un proceso sostenido de crecimiento económico deben asegurar que los procesos de captación de recursos del público en calidad de ahorro sean lo suficientemente eficaces para lograr que se conviertan en inversión. Aquí el papel fundamental lo deben desempeñar las instituciones financieras, por cuanto en la medida en que el desarrollo del mercado de capitales sea más débil, ese déficit de recursos deberá suplirse a través de procesos de consolidación del ahorro. Esto permite anticipar que el ahorro por sí solo no corresponde a una operación de inversión.

De otra parte, hablar de inversiones significa incorporar conceptos de rentabilidad. Para las operaciones de ahorro en el sistema financiero, la primera rentabilidad que aparece, corresponde a la rentabilidad bruta o rentabilidad facial que es igual a la tasa que ofrece la entidad financiera como atractivo para captar recursos del público. En el caso del CDT, se entiende como un ahorro del cual se espera un rendimiento económico, Álvarez (2013:201).

Por su parte, la rentabilidad neta es el resultado de incorporar en el rendimiento bruto los impuestos que se deben pagar por esos mismos rendimientos. En fin, la rentabilidad neta de una inversión es la rentabilidad efectiva corregida por los impuestos. Generalmente, a los rendimientos financieros (intereses) que se obtienen en una operación de ahorro se les aplica un mecanismo fiscal denominado retención en la fuente, que consiste en deducir de los intereses devengados un porcentaje, que pasa al fisco y se considera como un anticipo del pago de impuestos. Para el caso específico de los intereses, este porcentaje es del 7\%. Es evidente que esta deducción afecta el rendimiento sobre el dinero, porque al final de cuentas se reciben menos intereses que los esperados.

Por esta razón, cuando se realizan análisis de rendimientos no se puede desconocer el efecto que ejercen los impuestos (retención en la fuente) sobre la inversión. Al establecerse la relación entre los intereses netos recibidos (intereses devengados menos la retención en la fuente) y la inversión, resulta la rentabilidad neta, Meza (2008:184).

Así, la rentabilidad final o la que debe ser punto de referencia para la toma de decisiones de los inversionistas corresponde a la rentabilidad real, que para Meza (2008:184) concierne a la tasa de rendimiento o intereses que el mercado financiero está dispuesto a reconocerle a los ahorradores o inversionistas en ausencia de la inflación. Lo que significa que la tasa real es el rendimiento neto una vez descontada la inflación. 


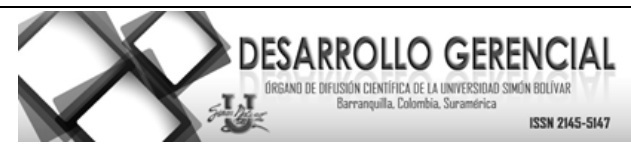

Es por ello que al analizar cualquier operación que involucre el manejo de dinero es necesario ver el efecto que produce la inflación; en consecuencia, la tasa real debe ser la preocupación permanente de todo inversionista que aspire a ver crecer su dinero en términos reales, ya que nada gana con obtener un rendimiento sobre una inversión si la inflación iguala o supera ese rendimiento, Meza (2008:186).

El proceso de bancarización en Colombia, que se viene dando por el consumo más que por el ahorro, deberá experimentar un giro contundente como resultado de la implementación de verdaderos incentivos al ahorro por parte de las entidades financieras; de lo contrario, los bajos niveles de profundización financiera seguirán en las mismas condiciones hasta tanto los instrumentos que se publicitan como mecanismo de ahorro y de inversión, garanticen el cumplimiento del objetivo para el cual se diseñaron.

Los bancos deberán replantear el sistema de fijación de sus tasas pasivas, e involucrar en su implementación el concepto de ahorro, inversión y rendimiento real, para garantizar que los certificados de depósito a término se adjudiquen en todos los plazos y sin discusión alguna como instrumento de ahorro y de inversión, aprovechando que se encuentran en una estructura especializada con sana competencia y compromiso con sus clientes, Toro et al (2012:35).

Todo proceso de inversión deberá ser medido por las tres rentabilidades básicas que definen la conveniencia o no de la aplicación de recursos, como son: la rentabilidad bruta, la rentabilidad neta y la rentabilidad real. En el caso de los certificados de depósito a término, la rentabilidad bruta o facial corresponde a los rendimientos que ofrecen las instituciones financieras y que en Colombia son ampliamente publicitadas. La rentabilidad neta resulta de involucrar el impuesto a los rendimientos financieros y la rentabilidad real resulta de contemplar la inflación sobre la rentabilidad neta. Incluso los mismos bancos que desean invertir en CDT deberán evaluar estas rentabilidades y tendrán que tener una calificación de riesgo para esta operación, Chavarro (2013:104).

Para determinar si efectivamente el certificado de depósito a término es un ahorro, los intereses que se reciben de las entidades financieras deberán garantizar el poder de compra de los recursos depositados, situación que en los depósitos a 30 y 60 días no se da. Por lo tanto, difícilmente podrá considerarse como ahorro ya que los recursos se deterioran en términos de su poder adquisitivo. Este aspecto se repite en la evaluación del título como instrumento de inversión, pues al no mantenerse el poder de compra tampoco se puede obtener rendimiento del mismo; por consiguiente, el certificado de depósito a término se convierte en instrumento no representativo de inversión, por más que la guerra de precios entre los bancos persista y las tasas varíen entre uno y otro.

Los plazos en los cuales se logra obtener algunos rendimientos reales corresponden a periodos de maduración por encima de los 60 días, un rango en el cual está por fuera la mayor proporción de potenciales ahorradores colombianos, lo que dificulta aún más las intenciones de que la mayor cantidad de

Desarrollo Gerencial, 7 (1) pp. 160 - 174 Enero-Junio 2015. ISSN: 2145-5147 (On Line). Universidad Simón Bolívar. 


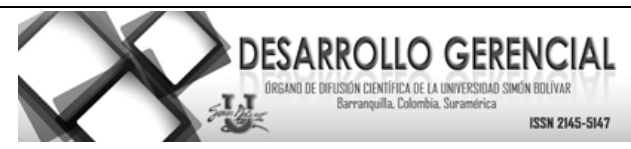

personas posean instrumentos de ahorro para lograr mayores niveles de bancarización en Colombia. Sin embargo, para Agudelo y Arango (2008:7): “los CDT se constituyen en el activo financiero más representativo del mercado de dineros en Colombia".

Se recomiendan como estudios complementarios, la evaluación del sistema de premios u obsequios de la banca para motivar a mayores niveles de captación y la determinación del costo real de las líneas de crédito del sistema financiero. Lo anterior permitirá integrar resultados para incentivar cambios trascendentales en los esquemas de fijación de tasas de interés y comisiones de estas instituciones.

\section{5. - REFERENCIAS}

Albea, D. (2002). Security Interests in Deposit Accounts and the banking Industry's use of set off. Alabama Law Review, 54(1).

Agudelo, D. \& Arango, M. (2008). La curva de rendimientos a plazo y las expectativas de tasas de interés en el mercado de renta fija en Colombia, 2002-2007. Lecturas de Economía, 68, 39. Recuperado de: http://search.proquest.com/docview/890085156?accountid=41931

Álvarez, M. J. (2013). Contratos mercantiles. Colombia: Editorial Universidad de Ibagué.

Arenas, R. (2004, diciembre 16). Opinión, ahorro e inversión para un crecimiento sostenido en Colombia.

Portafolio. Noticias Financieras. Recuperado de:

http://search.proquest.com/docview/468184939?accountid=41931

Becerra, O. R. \& Melo, L. F. (2009). Transmisión de tasas de interés bajo el esquema de metas de inflación: evidencia para Colombia. Cuadernos de Economía., 133(46), 108-26.

Bernal, J. A. H. (2012). Renta variable. Una alternativa de inversión en Colombia. Recuperado de: http://search.proquest.com/docview/1326257037?accountid=41931

Catica, B, J. R. \& Parra, A, M. P. (2012). La bancarización en Colombia frente al costo de los servicios financieros. 766-777. 7(2), Recuperado de: http://search.proquest.com/docview/1326257198?accountid=41931.

Caja de Crédito Agrario Industrial Y MINERO. (1967). Autosuperación integral por el ahorro. Bogotá: Editorial Los Mosqueteros.

Cano, C. et al. (2008). Mecanismo de transmisión de las tasas de interés en Colombia (2001-2007). Cuadernos de Economía No.27. 


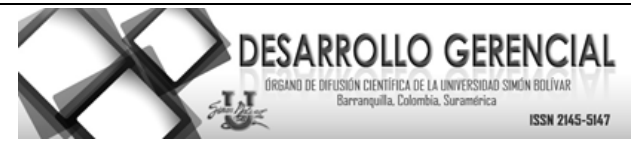

Cática, J. R., Parra, M. \& López, O. (2014). Retos de la banca colombiana para mejorar la profundización financiera. Revista Internacional Administración y Finanzas, 7(4), pag 27-37.

Chavarro, J. E. (2013). Estatuto Orgánico del Sistema Financiero Colombiano. Comentarios, concordancias, doctrina, jurisprudencia. Colombia: Nueva Legislación.

Kasilingam, R. \& Jayabal G. (2009). A Study on the Perception of Investors towards Small Saving Scheme. Paradig, 13(2), 1-12.

Melo, L. \& Becerra, O. (2006). Una aproximación a la dinámica de las tasas de interés de corto plazo en Colombia a través de modelos GARCH multivariados. Recuperado de: http://banrep.gov.co/docum/ftp/borra366.pdf

Meza, O. J. (2008). Matemáticas financieras aplicadas. Uso de las calculadoras Financieras y Prácticas en Excel. Bogotá: Editorial Ecoe Ediciones.

Muhleisen, M. (1997). Improving Indian's saving performance. Finance \& Development, June: 38 - 41.

Niculescu-aron, I. (2012). An Empirical analysis on preferred saving instruments based on the Romanian Households. Journal of Applied Quantative Methods, 7(4), 19-28.

Nokuman, K. (2002). The volatility of Japanese interest rates evidence for Certificate of Deposit and Gensaki rates. Internatioal Review of Financial Analysis, 11, 29-38.

Orazio, P. ET AL. (2000). Saving, growth, and investment: A macroeconomic analysys using panel of countries. The Review of Economics and Statistics, 82(2), 182-211.

Parra, I. (1976). El desarrollo y el ahorro personal. Bogotá: División de Artes Gráficas Departamento de Materiales de la Caja de Crédito Agrario Industrial y Minero.

Periódico Portafolio (2012). Depósito en CDT y cuentas de ahorro aumentaron 22\%. Tomado de: http://www.portafolio.co/economia/depositos-cdt-y-cuentas-ahorro-aumentaron-22

Poveda, R. G. (1968). Problemas del ahorro privado en Colombia. Medellín: Asociación Nacional de Industriales ANDI.

Toro, D. ET AL. (2012). Sistema financiero colombiano: evolución y perspectivas. Tesis de especialización no publicada, Universidad EAN, Armenia. 